\title{
The Effect of Periplaque Fat on Coronary Plaque Vulnerability in Patients with Stable Coronary Artery Disease - a 128-multislice CT-based Study
}

\author{
Nóra Raț1,2,3, Diana Opincariu1,2,3, Emese Márton2,3, Ramona Zavate2,3, Mirela Pintican²,3, \\ Theodora Benedek ${ }^{1,2,3}$ \\ ${ }^{1}$ University of Medicine and Pharmacy, Tîrgu Mureș, Romania \\ ${ }^{2}$ Center of Advanced Research in Multimodality Cardiac Imaging, Cardio Med Medical Center, Tîrgu Mureș, Romania \\ ${ }^{3}$ Cardiac Critical Care Unit, Clinic of Cardiology, County Clinical Emergency Hospital, Tîrgu Mureș, Romania
}

\section{CORRESPONDENCE}

\section{Diana Opincariu}

Str. Gheorghe Marinescu nr. 38

540139 Tîrgu Mures, Romania

Tel: +40 265215551

E-mail: diana.opincariu@yahoo.ro

\section{ARTICLE HISTORY}

Received: April 5, 2018

Accepted: June 17, 2018
Nóra Raț • Str. Gheorghe Marinescu nr. 38, 540139 Tîrgu Mureș, Romania. Tel: +40 265215 551, E-mail: ratnora@gmail.com

Emese Márton • Str. Gheorghe Marinescu nr. 50 540136 Tîrgu Mures, Romania. Tel: +40 265212 111, E-mail: emese marton92@gmail.com

Ramona Zavate • Str. Gheorghe Marinescu nr. 50 540136 Tîrgu Mures, Romania. Tel: +40 265212111 E-mail: ramona.zavate@gmail.com

Mirela Pintican - Str. Gheorghe Marinescu nr. 50 540136 Tîrgu Mures, Romania. Tel: +40 265212111 E-mail: evident.blessed@yahoo.com

Theodora Benedek • Str. Gheorghe Marinescu nr. 38 540139 Tîrgu Mures, Romania. Tel: +40 265215 551, E-mail: theodora.benedek@gmail.com

\begin{abstract}
Background: The role of periplaque fat (PPF), as a fragment of the total epicardial adipose tissue, measured in the vicinity of a target coronary lesion, more specifically within the close proximity of a vulnerable plaque, has yet to be evaluated. The study aimed to evaluate the interrelation between PPF and coronary plaque vulnerability in patients with stable coronary artery disease (CAD). Secondary objective: evaluation of the relationship between the total pericardial fat and markers for plaque vulnerability. Materials and methods: We prospectively enrolled 77 patients with stable CAD, who underwent 128-multislice computed tomography coronary angiography (CTCA), and who presented minimum one lesion with $>50 \%$ stenosis. CTCA analysis included measurements of total pericardial fat and PPF volumes, coronary plaque characteristics, markers for plaque vulnerability - positive remodeling (PR), low attenuation plaque (LAP), spotty calcifications (SC,) napkin ring sign (NRS). Study subjects were divided into two categories: Group 1 - 1 marker of plaque vulnerability ( $n=36,46.75 \%$ ) and Group $2-\geq 1$ marker of vulnerability $(n=41,53.25 \%)$. Results: The mean age of the population was $61.77 \pm 11.28$ years, and 41 (53.24\%) were males. The analysis of plaque characteristics showed that Group 2 presented significantly longer plaques (16.26 $\pm 4.605 \mathrm{~mm}$ vs. $19.09 \pm 5.227 \mathrm{~mm}, p=0.02$ ), remodeling index (0.96 \pm 0.20 vs. $1.18 \pm 0.33, p=0.0009$ ), and vessel volume $(p=0.027)$, and more voluminous plaques $\left(147.5 \pm 71.74 \mathrm{~mm}^{3} \mathrm{vs} .207 .7 \pm 108.9 \mathrm{~mm}^{3}\right.$ $p=0.006)$ compared to Group 1. Group 2 presented larger volumes of PPF $\left(512.2 \pm 289.9 \mathrm{~mm}^{3} \mathrm{vs}\right.$ $\left.710.9 \pm 361.9 \mathrm{~mm}^{3}, \mathrm{p}=0.01\right)$ and of thoracic fat volume $\left(1,616 \pm 614.8 \mathrm{~mm}^{3} \mathrm{vs} .2,000 \pm 850.9 \mathrm{~mm}^{3}\right.$, $p=0.02)$, compared to Group 1, but no differences were found regarding the total pericardial fat $(p=0.49)$. Patients with 3 or 4 vulnerability markers $(V M)$ presented significantly larges PPF volumes compared to those with 1 or $2 \mathrm{VM}$, respectively $(p=0.008)$. There was a significant positive correlation between PPF volume and the non-calcified $(r=0.474,95 \% \mathrm{Cl} 0.2797-0.6311$ $\mathrm{p}<0.0001)$, lipid-rich ( $r=0.316,95 \% \mathrm{Cl} 0.099-0.504, p=0.005)$, and fibro-fatty $(r=0.452,95 \% \mathrm{Cl}$ $0.2541-0.6142, p<0.0001$ ) volumes. The total pericardial fat was significantly correlated only with the volume of lipid-rich plaques $(p=0.02)$. Conclusions: Periplaque fat volume was associated with a higher degree of coronary plaque vulnerability. PPF was correlated with lipid-rich, fibro-fatty, and non-calcified plaque-related volumes, as markers for enhanced plaque vulnerability. PPF volume, assessed with native cardiac computed tomography, could become a novel marker for coronary plaque vulnerability.
\end{abstract}

Keywords: periplaque fat volume, vulnerable coronary plaques, coronary artery disease, pericardial fat volume, CT coronary angiography 


\section{BACKGROUND}

\section{Vulnerable coronary plaques}

Unstable coronary atheromas present increased risk for rupture, erosion, and thrombosis, with the consequent development of an acute coronary syndrome. The concept of "plaque vulnerability" refers to lesions that are prone to cause an acute event, which have been described as presenting a thin fibrous cap and underlying large lipid core, that leads to eccentric vascular remodeling followed by luminal narrowing and distal myocardial ischemia. ${ }^{1-3}$ Several invasive and noninvasive imaging methods have been described for the assessment of high-risk vulnerable coronary lesions. ${ }^{4-7}$ Invasive imaging techniques used for the identification of vulnerability markers (VM) for coronary atherosclerosis include intravascular ultrasound (with description of plaque burden, remodeling index, cross sectional area, and necrotic core,), and optical coherence tomography (which measures the thickness of the fibrous arch, lipid arch, intracoronary thrombi, or macrophages). 8,9 Computed tomography coronary angiography (CTCA) is a noninvasive method that provides visualization of the entire coronary tree, as well as the vessel lumen and wall characteristics, while offering similar accuracy in detecting coronary vulnerability as the intracoronary imaging techniques. ${ }^{10-12} \mathrm{VM}$ for coronary plaques assessed via CTCA include the presence and size of the lipid necrotic core, low attenuation plaques (LAP, density of $<30$ Hounsfield units), positive vessel remodeling (PR), the napkin ring sign (NRS) and spotty calcifications (SC). ${ }^{13-17}$

\section{Epicardial fat on severity of CAD and plaque vulnerability}

The total epicardial fat (EF) has been shown to be associated with the total coronary atherosclerotic burden, with the severity of coronary stenoses, and with cardiovascular risk factors. Moreover, EF is directly proportional with the rate of major adverse cardiac events in patients with known coronary artery disease. ${ }^{18-22}$ The epicardial adipose tissue is measured with either transthoracic echocardiography as linear thickness, or with volumetric assessment via cardiac computed tomography or magnetic resonance imaging. ${ }^{23}$ Recent studies connected the epicardial adipose tissue with the presence of high-risk coronary plaques, defined as presenting one of the following features: low attenuation, positive remodeling, napkin ring sign, spotty calcifications; studies have also shown that patients with high-risk plaques present a larger volume of epicardial fat, compared to those with no high-risk plaques..$^{24,25}$

\section{Pericoronary and periplaque fat}

Pericoronary adipose tissue, as a fragment of the total epicardial fat, is located in direct contact to the coronary artery wall and has pro-inflammatory properties through its paracrine effect and local release of inflammatory cytokines, which can trigger plaque formation, progression, vulnerabilization, and even rupture. ${ }^{26}$ Pericoronary fat (PF) has also been associated with the presence of vulnerable coronary plaques, even after adjustment for obesity and smoking. ${ }^{27}$ The ratio between PF and overall EF has been linked with a higher coronary plaque volume and an increased volume of mixed and non-calcified plaques. ${ }^{28,29}$ The role of periplaque fat (PPF), measured in the vicinity of a target coronary lesion, more specifically within the close proximity of a vulnerable plaque, has yet to be evaluated. The alternative hypothesis of the present study is that an increased volume of periplaque adipose tissue, measured adjacent to a hemodynamically significant coronary lesion, is associated with a more extensive plaque vulnerability (presence of plaque vulnerability features), as assessed with the use of CTCA.

The aim of the study was to evaluate the interrelation between the periplaque adipose tissue, measured in the vicinity of a significant coronary lesion, and the degree of plaque vulnerability in patients with stable coronary artery disease, who undergo noninvasive CTCA. Secondary objectives include the evaluation of the relationship between total pericardial fat and markers for plaque vulnerability.

\section{MATERIALS AND METHODS}

We conducted a prospective observational cohort study, which included 77 subjects who presented in outpatient settings, at the Cardio Med Medical Center, for symptoms indicative for coronary artery disease (chest pain, dyspnea, fatigue). All subjects underwent a complete clinical examination, 12-lead ECG tracing, two-dimensional transthoracic echocardiography, followed by 128-multislice CTCA. All patients who presented minimum one lesion with $\geq 50 \%$ stenosis were enrolled in the study.

Study procedures were conducted according to the ethical principles stated in the Declaration of Helsinki, and all subjects signed a written informed consent prior to being enrolled in the study.

Subjects with high clinical probability for presenting an acute coronary syndrome (unstable angina, acute ST- or non-ST-segment elevation myocardial infarction) 
TABLE 1. Patient characteristics

\begin{tabular}{lccc}
\hline & Group 1 & Group 2 & p value \\
\hline Age (years) & $62.14 \pm 10.97$ & $61.41 \pm 11.59$ & 0.7791 \\
Male gender, n (\%) & $26(72.22 \%)$ & $15(34.09 \%)$ & 0.0007 \\
Risk factors & & & \\
Hypertension (n, \%) & $28(68.09 \%)$ & $35(83.72 \%)$ & 0.13 \\
Hypertension, n (\%) & $29(80.56 \%)$ & $29(70.73 \%)$ & 0.4637 \\
Diabetes, n (\%) & $8(23.53 \%)$ & $14(34.15 \%)$ & 0.4529 \\
Peripheral arterial disease, n (\%) & $6(17.65 \%)$ & $5(12.20 \%)$ & 0.7364 \\
Acute myocardial infarction, n (\%) & $11(32.35 \%)$ & $12(29.27 \%)$ & 0.7730 \\
Smoker, n (\%) & $21(61.76 \%)$ & $23(56.10 \%)$ & 0.6198 \\
\hline
\end{tabular}

were excluded, as they were referred for emergency invasive coronary angiography. Other exclusion criteria were acute renal failure or terminal-stage chronic kidney disease, pregnancy or lactation, allergy to iodine contrast substance, active malignancy, or refusal to provide written informed consent for study enrollment.

CTCAs were performed with a Somatom Definition 128-Slice CT equipment (Siemens Healthcare GmbH, Ernlagen, Germany), and image acquisitions were analyzed for the following parameters: the overall epicardial adipose tissue volume, the adipose tissue located in the vicinity of the target coronary lesion (from herein referred to as periplaque fat - PPF), plaque-related features (markers for plaque vulnerability, length, degree of stenosis, volume, plaque components - necrotic core, fibro-fatty, fibrotic, and densely calcified volumes), vascular indices (remodeling index, eccentricity index).

The study population was divided into two groups, based on the presence of CT markers for plaque vulnerability (low attenuation plaque, positive remodeling, spotty calcification, napkin ring sign), as follows: Group 1 - patients with 1 marker of plaque instability $(\mathrm{n}=36)$; Group 2 - patients with $\geq 1$ marker of instability $(n=41)$.

Statistical analysis was performed using GraphPad Prism 7 software (GraphPad Software, Inc., San Diego, USA), with the application of two-tailed statistical comparative testing for unpaired continuous data, or Chi square test for categorical variables, at a statistical significance of alpha 0.05 . Spearman or Pearson coefficient when appropriate, was used to describe correlation analysis.

\section{RESULTS}

From the 77 study subjects, 36 (46.75\%) presented only 1 marker (Group 1), and 41 (53.24\%) presented more than 1 CT marker for plaque instability. The mean age of the overall study population was $61.77 \pm 11.28$ years, and 41 (53.24\%) subjects were males.

There were no significant differences between groups in relation to age ( $p=0.779)$, or cardiovascular risk factors including arterial hypertension $(\mathrm{p}=0.463)$, diabetes $(p=0.452)$, peripheral artery disease $(p=0.736)$, previous myocardial infarction ( $\mathrm{p}=0.773)$, or chronic tobacco use $(p=0.619)$ (Table 1$)$. However, patients in Group 1 presented a significantly higher number of male subjects, compared to Group 2 ( $\mathrm{p}=0.0007)$.

The distribution of CT markers for coronary plaque vulnerability is listed in Table 2 . In the first group, while there were no napkin ring signs found in the analyzed plaques, the most frequent vulnerability marker was the presence of spotty calcifications (52.78\% of cases). In the second group however, the most encountered vulnerability feature was positive vascular remodeling (in $73.81 \%$ of analyzed plaques) (Table 2).

TABLE 2. Presence of vulnerability features in the study groups

\begin{tabular}{lccc}
\hline & $\begin{array}{c}\text { Group 1 } \\
\mathbf{n = 3 6}\end{array}$ & $\begin{array}{c}\text { Group 2 } \\
\mathbf{n = ~ 4 1}\end{array}$ & p value \\
\hline Positive remodeling, $\mathrm{n}(\%)$ & $11(30.55 \%)$ & $31(75.60 \%)$ & 0.0001 \\
Spotty calcification, $\mathrm{n}(\%)$ & $19(52.78 \%)$ & $30(73.17 \%)$ & 0.3102 \\
Napkin ring sign, $\mathrm{n}(\%)$ & $0(0 \%)$ & $10(24.39 \%)$ & 0.0013 \\
Low attenuation, $\mathrm{n}(\%)$ & $6(16.67 \%)$ & $19(46.64 \%)$ & 0.0114 \\
\hline
\end{tabular}


TABLE 3. CT analysis of plaque characteristics in the study groups

\begin{tabular}{|c|c|c|c|c|}
\hline Parameter & $\begin{array}{c}\text { All } \\
\mathrm{n}=77\end{array}$ & $\begin{array}{c}\text { Group } 1 \\
1 \text { vulnerability } \\
\text { marker } \\
n=36\end{array}$ & $\begin{array}{c}\text { Group } 2 \\
>1 \text { vulnerability } \\
\text { marker } \\
n=\mathbf{4 1}\end{array}$ & $\mathrm{p}$ value \\
\hline Plaque length, mm & $17.76 \pm 5.115$ & $16.26 \pm 4.605$ & $19.09 \pm 5.227$ & 0.0214 \\
\hline Stenosis, \% & $56.57 \pm 11.25$ & $54.03 \pm 10.06$ & $58.8 \pm 11.88$ & 0.0575 \\
\hline Minimum luminal area, mm² & $0.041 \pm 0.026$ & $0.047 \pm 0.025$ & $0.036 \pm 0.026$ & 0.0543 \\
\hline Eccentricity index & $0.36 \pm 0.22$ & $0.374 \pm 0.239$ & $0.347 \pm 0.219$ & 0.6604 \\
\hline Remodeling index & $1.079 \pm 0.298$ & $0.9619 \pm 0.200$ & $1.182 \pm 0.333$ & 0.0009 \\
\hline Vessel volume, $\mathrm{mm}^{3}$ & $297 \pm 139.1$ & $256.2 \pm 115.9$ & $332.8 \pm 148.9$ & 0.0272 \\
\hline Lumen volume, $\mathrm{mm}^{3}$ & $117.4 \pm 64.97$ & $108.6 \pm 57.06$ & $125 \pm 70.61$ & 0.4250 \\
\hline Plaque volume, $\mathrm{mm}^{3}$ & $179.6 \pm 97.6$ & $147.5 \pm 71.74$ & $207.7 \pm 108.9$ & 0.0061 \\
\hline Calcified volume, $\mathrm{mm}^{3}$ & $30.92 \pm 37.69$ & $34.2 \pm 44.57$ & $28.05 \pm 30.7$ & 0.9091 \\
\hline Calcified, \% & $18.52 \pm 21.98$ & $21.1 \pm 24.67$ & $16.26 \pm 19.35$ & 0.3377 \\
\hline Non-calcified volume, $\mathrm{mm}^{3}$ & $148.7 \pm 95.02$ & $113.3 \pm 64.2$ & $179.8 \pm 106.9$ & 0.0017 \\
\hline Non-calcified, \% & $81.49 \pm 21.97$ & $78.91 \pm 24.64$ & $83.75 \pm 19.35$ & 0.4032 \\
\hline Lipid-rich volume, $\mathrm{mm}^{3}$ & $11.49 \pm 17.66$ & $5.494 \pm 6.352$ & $16.75 \pm 22.28$ & 0.0128 \\
\hline Lipid-rich, \% & $5.71 \pm 7.33$ & $3.781 \pm 3.887$ & $7.405 \pm 9.093$ & 0.0661 \\
\hline Fibrotic volume, $\mathrm{mm}^{3}$ & $137.2 \pm 87.22$ & $107.8 \pm 60.67$ & $163 \pm 98.87$ & 0.0049 \\
\hline Fibrotic, \% & $75.77 \pm 20.91$ & $75.13 \pm 23.6$ & $76.34 \pm 18.52$ & 0.7472 \\
\hline
\end{tabular}

\section{CT analysis of plaque-related features}

When analyzing the CT characteristics of the target coronary plaques, we found no statistically significant differences between the two groups regarding the degree of vascular stenosis $(p=0.057)$, minimum lumen area $(\mathrm{p}=0.054)$, eccentricity index $(\mathrm{p}=0.660)$, lumen volume $(\mathrm{p}=0.425)$, or other plaque related volumes (Table 3$)$. However, the results showed significantly longer plaques in patients from Group 2, with more than one vulnerability feature $(\mathrm{p}=0.021)$, a significantly higher remodeling index $(0.96 \pm 0.20$ vs. $1.18 \pm 0.33, p=0.0009)$ and vessel volume $(\mathrm{p}=0.027)$, as well as significantly more volumi- nous plaques $\left(147.5 \pm 71.74 \mathrm{~mm}^{3}\right.$ vs. $207.7 \pm 108.9 \mathrm{~mm}^{3}$, $\mathrm{p}=0.006$ ). The analysis of plaque-related volumes revealed that patients in Group 2 presented significantly larger noncalcified volumes $(\mathrm{p}=0.001)$, larger lipid-rich volumes ( $\mathrm{p}$ $=0.012)$, and larger fibrotic volumes $(\mathrm{p}=0.004)$ in the analyzed coronary plaques (Table 3 ).

\section{Periplaque fat, total pericardial fat, and thoracic fat in coronary vulnerability}

Patients with more than one marker for coronary vulnerability (Group 2), presented a significantly larger volume
Periplaque fat volume

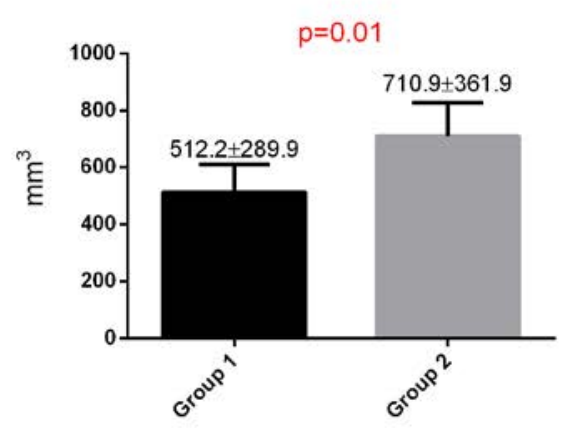

A
Total pericardial fat volume

$p=0.49$

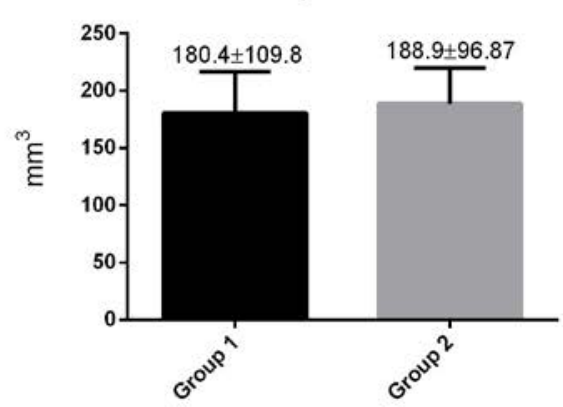

B

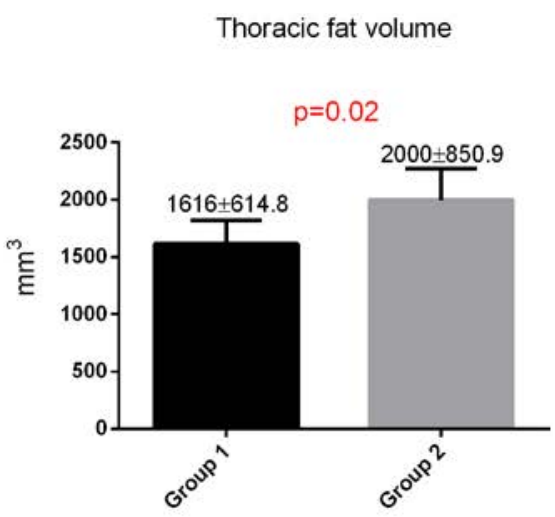

C

FIGURE 1. Comparative analysis between the two coronary vulnerability groups. A - Periplaque fat volume; B - Total Pericardial fat volume; $\mathbf{C}$ - Thoracic fat volume 


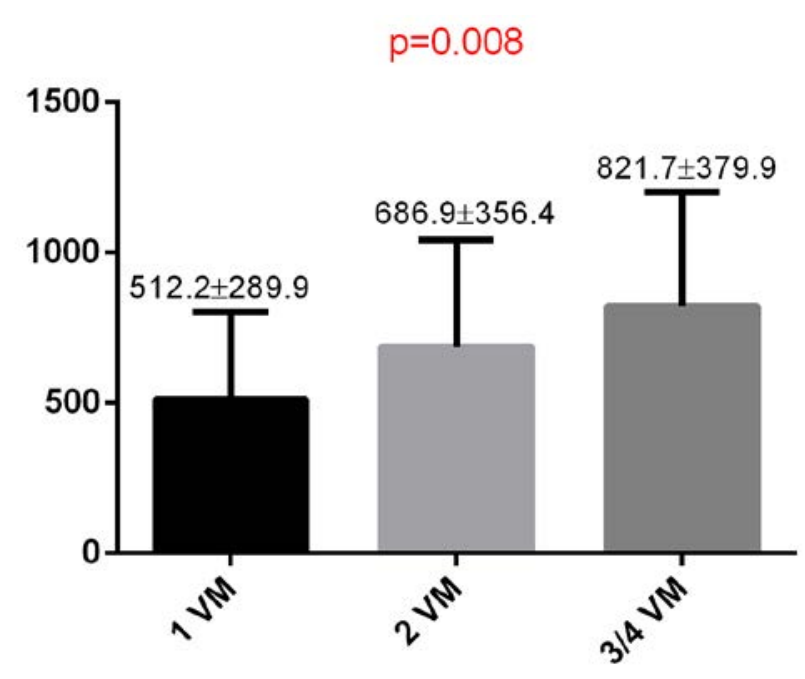

FIGURE 2. Periplaque fat $\left(\mathrm{mm}^{3}\right)$ and vulnerability degree of coronary plaques. VM - vulnerability markers

of periplaque fat (PPF) compared to Group 1 (Figure 1A). No significant differences were recorded between the two groups regarding the total pericardial fat volume (Figure $1 B)$, but the thoracic fat volume was significantly larger in Group $2(\mathrm{p}=0.02)$ (Figure 1C).
The analysis of periplaque volume based on the vulnerability degree of the coronary plaques showed that patients with 3/4 CT markers for plaque instability presented a significantly higher volume of PPF compared to those presenting one or two markers, respectively $(\mathrm{p}=0.008)$ (Figure 2).

\section{Correlations between periplaque fat, total pericardial fat and plaque related volumes}

Linear regression analysis revealed significant positive correlations between the volume of adipose tissue located around the analyzed plaque and the following plaquerelated volumes: non-calcified volume $(r=0.474,95 \% \mathrm{CI}$ $0.2797-0.6311, \mathrm{p}<0.0001)$, lipid-rich volume $(\mathrm{r}=0.316$, 95\% CI $0.099-0.504, \mathrm{p}=0.005)$, and fibro-fatty volume $(\mathrm{r}=0.452,95 \%$ CI $0.2541-0.6142, \mathrm{p}<0.0001)$ (Figure 3 ). However, there was no significant correlation between the periplaque volume and the total plaque volume $(\mathrm{r}=-0.12$, $95 \%$ CI $-0.3475-0.1063, \mathrm{p}=0.27)$ or the calcified volume $(\mathrm{p}=0.6)$ (Figure 3$)$.

The total pericardial fat was not significantly correlated with the plaque volume $(\mathrm{p}=0.4)$, calcified volume

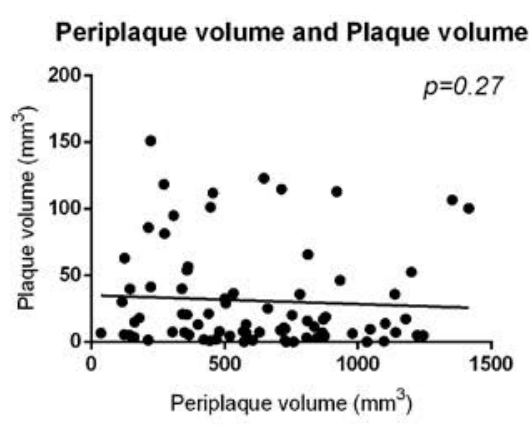

A

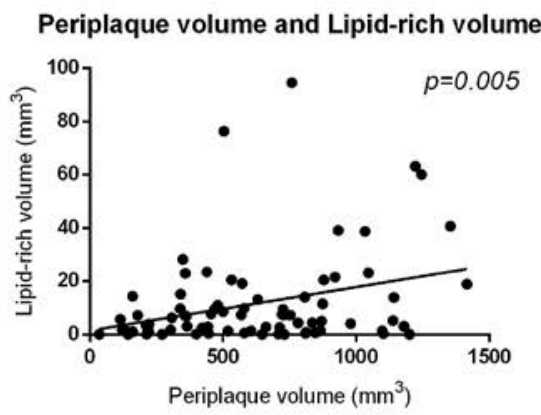

D

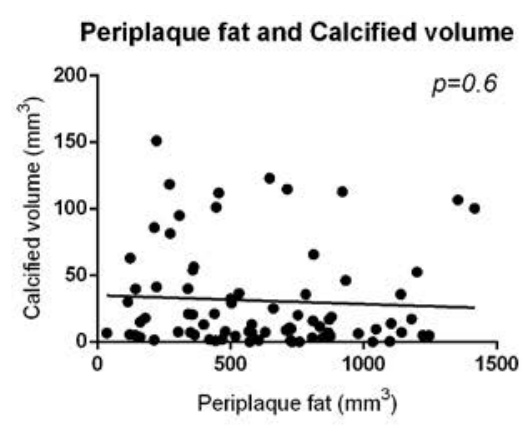

B

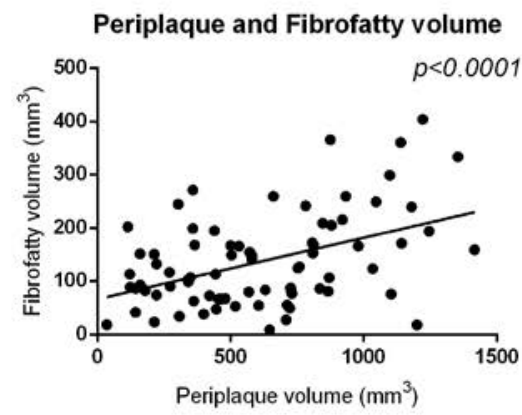

E

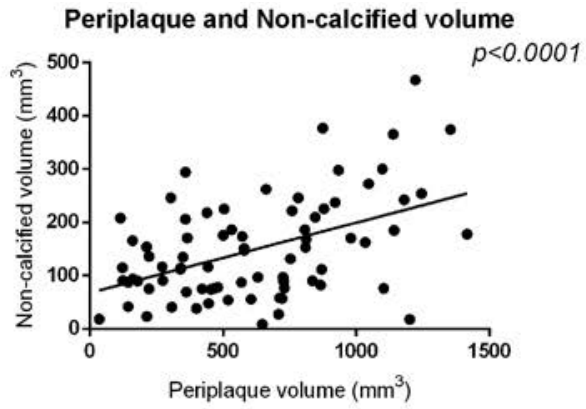

C

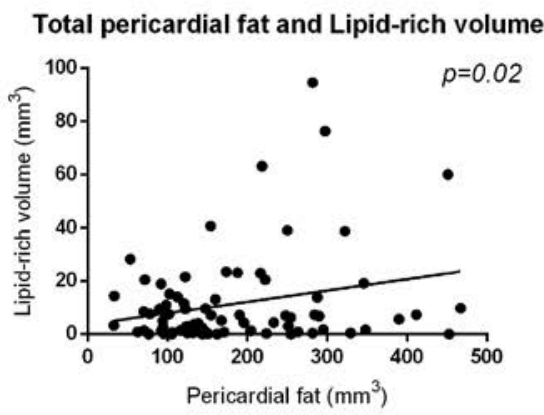

$\mathbf{F}$

FIGURE 3. Correlations between periplaque fat volume and plaque volume (A), calcified plaque volume (B), non-calcified plaque volume (C), lipid-rich volume (D), fibro-fatty volume (E), and total pericardial fat and lipid-rich volume (F). 
$(\mathrm{p}=0.6)$, non-calcified plaque volume $(\mathrm{p}=0.2)$, or fibrofatty volume $(p=0.4)$. However, the lipid-rich volume plaques were significantly correlated with the total volume of the pericardial fat $(\mathrm{p}=0.02)$ (Figure $3 \mathrm{~F})$.

\section{DISCUSSIONS}

The present study aimed to evaluate the relationship between the periplaque fat volume and the presence of CT vulnerability markers for coronary plaques. The adipose tissue surrounding the heart, more specifically the epicardial and pericardial fat, has been the subject of numerous studies that included coronary artery disease patients. ${ }^{18,30}$ Epicardial fat represents the visceral adipose tissue between the myocardial cells and the visceral layer of the pericardial membrane, while the pericoronary or perivascular adipose tissue is observed in the vicinity of the coronary vessels. Moreover, the pericardial fat, which is similar to the subcutaneous adipose tissue, having also a common embryological origin, is located between the two layers of the pericardial membrane. ${ }^{31}$

Noninvasive evaluation of epicardial adipose tissue is performed with the help of 2D transthoracic echocardiography as a hypoechoic space at the level of the free wall of the right ventricle during diastole, with non-contrast multidetector computed tomography, which can assess either thickness or volume of the overall EF or in various segments, such as pericoronary or periplaque, as performed in the present study. The gold-standard imaging technique for epicardial fat evaluation is, however, cardiac magnetic resonance imaging, which presents higher costs and low availability, therefore having limited use. ${ }^{32-34}$

Both the pericardial and epicardial adipose tissue have been shown to be correlated with the overall increased cardiovascular risk, as well as with the presence of high-risk coronary plaques. ${ }^{35,36}$ Pericoronary adipose tissue has recently emerged as a subject of interest in studies on novel cardiovascular risk factors and on promotors for coronary atherosclerosis and vulnerability. ${ }^{37}$ Several studies on histopathological samples of epicardial adipose tissue have revealed that EF contains several pro-inflammatory mediators, which can contribute to the extravascular causes of atherosclerosis progression. ${ }^{38-40}$

The present study found that patients with significant coronary atherosclerosis, who presented high-risk coronary plaques, with more than one CT plaque vulnerability marker, presented significantly higher volumes of periplaque fat and thoracic fat volume compared to patients with one CT vulnerability marker (either positive remodeling, spotty calcifications, low attenuation plaque, or napkin ring sign). Despite the previously mentioned findings, we found no significant association between a higher degree of plaque vulnerability and the total volume of the pericardial adipose tissue. A subanalysis from the Framingham heart study showed that pericardial fat and visceral abdominal fat were independently associated with measures of obesity (waist circumference, body mass index) as cardiovascular risk factors, but the association was not valid after adjustments for traditional risk factors. ${ }^{35}$

In addition, when evaluating the volume of epicardial adipose tissue measured at the level of the target coronary plaque based on an increasing degree of coronary plaque vulnerability, we found that subjects with three or four CT vulnerability markers presented a significantly higher PPF volume compared to those with two or one vulnerability marker, respectively.

Although periplaque fat has not been studied, Hassan et al. sought to evaluate the relationship between the segmental epicardial fat volume, evaluated with the use of cardiac magnetic resonance (CMR), and the underlying coronary plaque characteristics in patients with stable angina. Their study included the evaluation of each coronary artery segment $(n=8)$ with the assessment of segmental adipose tissue volume (with CMR) and the evaluation of coronary plaque features via multidetector computed tomography. Their results revealed a significant correlation between segmental epicardial fat volume and increasing luminal stenosis $(\mathrm{p}<0.001)$, and lesions with mixed plaques and low-attenuation non-calcified plaques presented significantly greater volumes of segmental epicardial fat volumes compared to lesions with calcified or CT attenuation plaques. ${ }^{25}$

Our study found significant linear correlations between periplaque fat volume and several plaque-related volumes, which indicate a propensity towards higher vulnerability, including the non-calcified, lipid-rich, and fibro-fatty volumes. This finding suggests that a larger adipose tissue located near a hemodynamically significant coronary lesion is correlated with increased volumes of soft plaque components, which offers a higher degree of lesion instability. On the other hand, no significant correlations were found between the total plaque volume and the volume of calcified components within the analyzed plaque, and a higher extension of periplaque adipose tissue.

\section{CONCLUSIONS}

Epicardial adipose tissue located in the vicinity of hemodynamically significant coronary stenosis was associated with a higher degree of plaque vulnerability, as assessed 
with cardiac computed tomography. Periplaque fat volume was significantly correlated with lipid-rich, fibro-fatty, and non-calcified plaque-related volumes, as markers for enhanced plaque vulnerability.

Pericardial fat volume was not significantly associated with a higher vulnerability degree of the analyzed coronary plaque, but it was significantly correlated with the lipid-rich volume.

Periplaque fat volume, assessed with native cardiac computed tomography, could become a novel marker for coronary plaque vulnerability, but further larger studies on obstructive coronary artery disease are required.

\section{CONFLICT OF INTEREST}

Nothing to declare.

\section{ACKNOWLEDGEMENT}

The study is part of the PhD study entitled "New imaging markers in cardiovascular disease" financed by the program of doctoral research in the University of Medicine and Pharmacy of Tîrgu Mureș, Romania, contract number $14057 / 38$ from $07 / 10 / 2014$.

\section{REFERENCES}

1. Stefanidis C, Antoniou C, Tsiachris D, Pietri P. Coronary Atherosclerotic Vulnerable Plaque: Current Perspectives. J Am Heart Assoc. 2017:6:e005543

2. Schaar JA, Muller JE, Falk E, et al. Terminology for high-risk and vulnerable coronary artery plaques. Eur Heart J. 2004;25:1077-1082.

3. Varnava AM, Mills PG, Davies MJ. Relationship between coronary artery remodeling and plaque vulnerability. Circulation. 2002;105:939-943,

4. Nyulas T, Chitu M, Mester A, et al. Computed Tomography Biomarkers of Vulnerable Coronary Plaques. Journal of Interdisciplinary Medicine. 2016:1:263-266

5. Opolski MP, Kępka C, Rużyłło W. Computed tomography for detection o vulnerable coronary plaque - A Cassandra's dream? Postępy w Kardiologii Interwencyjnej/Advances in Interventional Cardiology. 2014;10:147-152.

6. Motoyama S, Masayoshi S, Harigaya H, et al. Computed tomographic angiography characteristics of atherosclerotic plaques subsequently resulting in acute coronary syndrome. J Am Coll Cardiol. 2009;54:49-57.

7. Motoyama S, Kondo T, Sarai M, et al. Multislice computed tomographic characteristics of coronary lesions in acute coronary syndromes. J Am Coll Cardiol. 2007:50:319-326.

8. Maurovich-Horváth P, Merkely B. Intracoronary Imaging and Plaque Vulnerability. Journal of Cardiovascular Emergencies. 2016:2:148-150.

9. Benedek T, Mester A, Benedek A, Rat N, Opincariu D, Chițu M. Assessment of Coronary Plaque Vulnerability in Acute Coronary Syndromes using Optical Coherence Tomography or Intravascular Ultrasound. A systematic Review. Journal of Cardiovascular Emergencies. 2016;2:173-184

10. Rodriguez-Granillo GA, Carrascosa P, Bruining N, Waksman R, GarciaGarcia HM. Defining the non-vulnerable and vulnerable patients with computed tomography coronary angiography: evaluation of atherosclerotic plaque burden and composition. Eur Heart J Cardiovasc Imaging. 2016:17:481-491.

11. Voros S, Rinehart S, Qian Z, et al. Coronary atherosclerosis imaging by coronary CT angiography: current status, correlation with intravascular interrogation and meta- analysis. JACC Cardiovasc Imaging. 2011;4:537548.
12. Benedek T, Gyöngyösi M, Benedek I. Multislice computed tomographic coronary angiography for quantitative assessment of culprit lesions in acute coronary syndromes. Can J Cardiol. 2013;29:364-371.

13. Choi BJ, Kang DK, Tahk SJ, et al. Comparison of 64-slice multidetector computed tomography with spectral analysis of intravascular ultrasound backscatter signals for characterizations of noncalcified coronary arteria plaques. Am J Cardiol. 2008;102:988-993.

14. Kroener E, van Velzen J, Boogers M, et al. Positive remodeling on coronary computed tomography as a marker for plaque vulnerability on virtual histology intravascular ultrasound. Am J Cardiol. 2011;107:1725-1729.

15. Maurovich-Horvat P, Schlett $\mathrm{CL}$, Alkadhi $\mathrm{H}$, et al. The napkin-ring sign indicates advanced atherosclerotic lesions in coronary CT angiography. JACC Cardiovasc Imaging. 2012;5:1243-1252.

16. Thomsen C, Abdulla J. Characteristics of high-risk coronary plaques identified by computed tomographic angiography and associated prognosis: a systematic review and meta-analysis. Eur Heart J Cardiovasc Imaging. 2016;17:120-129

17. Hou ZH, Lu B, Gao Y, et al. Prognostic value of coronary CT angiography and calcium score for major adverse cardiac events in outpatients. JACC Cardiovasc Imaging. 2012;5:990-999.

18. Benedek T, Opincariu D, Rat N, Hodas R, Mester A, Benedek I. The Assessment of Epicardial Adipose Tissue in Acute Coronary Syndrome Patients. A Systematic Review. Journal of Cardiovascular Emergencies. 2017:3:18-29.

19. Gitsioudis G, Schmahl C, Missiou A, et al. Epicardial adipose tissue is associated with plaque burden and composition and provides incremental value for the prediction of cardiac outcome. A clinical cardiac computed tomography angiography study. PLoS One. 2016;11:e0155120.

20. Nakanishi R, Rajani R, Cheng VY, et al. Increase in epicardial fat volume is associated with greater coronary artery calcification progression in subjects at intermediate risk by coronary calcium score: a serial study using non-contrast cardiac CT. Atherosclerosis. 2011;218:363-368.

21. Mahabadi A, Berg M, Lehmann N, et al. Association of Epicardial Fat With Cardiovascular Risk Factors and Incident Myocardial Infarction in the General Population: The Heinz Nixdorf Recall Study. Journal of the American College of Cardiology. 2013:61:1388-1395.

22. Fukamachi D, Higuchi Y, Hiro T, et al. Association between the epicardial adipose tissue thickness and the presence of multivessel disease in patients with acute myocardial infarction. Journal of Atherosclerosis and Atherothrombosis. 2014;2:144-151.

23. Xu Y, Cheng $X$, Hong K, Huang C, Wan L. How to interpret epicardial adipose tissue as a cause of coronary artery disease: a meta-analysis. Coron Artery Dis. 2012;23:227-233.

24. Nerleklar N, Brown AJ, Muthalaly RG, et al. Association of Epicardial Adipose Tissue and High-Risk Plaque Characteristics: A Systemic Review and Meta-Analysis. Journal of American Heart Association. 2017;6:e006379.

25. Hassan M, Said K, Rizk $H$, et al. Segmental peri-coronary epicardial adipose tissue volume and coronary plaque characteristics. European Heart Journal-Cardiovascular Imaging. 2016;17:1169-1177.

26. Maurovich-Horvat P, Kallianos K, Engel LC, et al. Influence of pericoronary adipose tissue on local coronary atherosclerosis as assessed by a novel MDCT volumetric method. Atherosclerosis. 2011:219:151-157.

27. Okubo R, Nakanishi R, Toda M, et al. Pericoronary adipose tissue ratio is a stronger associated factor of plaque vulnerability than epicardial adipose tissue on coronary computed tomography angiography. Heart Vessels. 2017;32:813-822

28. Mahabadi AA, Reinsch N, Lehmann N, et al. Association of pericoronary fat volume with atherosclerotic plaque burden in the underlying coronary artery: a segment analysis. Atherosclerosis. 2010:211:195-199.

29. Gorter PM, van Lindert AS, de Vos AM, et al. Quantification of epicardial and pericoronary fat using cardiac computed tomography; reproducibility and relation with obesity and metabolic syndrome in patients suspected of coronary artery disease. Atherosclerosis. 2008;197:896-903.

30. Sinha SK, Thakur R, Jha MJ, et al. Epicardial Adipose Tissue Thickness and Its Association With the Presence and Severity of Coronary Artery Disease in Clinical Setting: A Cross-Sectional Observational Study. Journal of Clinical Medicine Research. 2016;8:410-419.

31. lacobellis G. Epicardial and pericardial fat: close, but very different. Obesity (Silver Spring). 2009;17:625.

32. Bertaso A, Bertol D, Duncan B, Foppa M. Epicardial Fat: Definition, Measurements, and Systematic Review of Main Outcomes. Arq Bras Cardiol. 2013;101:18-28.

33. Machann J, Thamer C, Schnoedt B, et al. Standardized assessment of whole body adipose tissue topography by MRI. J Magn Reson Imaging. 2005:21:455-62. 
34. Sarin S, Wenger C, Marwaha A, et al. Clinical significance of epicardial fat measured using cardiac multislice computed tomography. Am J Cardiol. 2008:102:767-771.

35. Mahabadi AA, Massaro JM, Rosito GA, et al. Association of pericardial fat, intrathoracic fat, and visceral abdominal fat with cardiovascular disease burden: the Framingham Heart Study. Eur Heart J. 2009;30:850-856.

36. Ding J, Hsu FC, Harris TB, et al. The association of pericardial fat with incident coronary heart disease: the Multi-Ethnic Study of Atherosclerosis (MESA). Am J Clin Nutr. 2009;90:499-504.

37. Gorter PM, de Vos AM, van der Graaf $Y$, et al. Relation of epicardial and pericoronary fat to coronary atherosclerosis and coronary artery calcium in patients undergoing coronary angiography. Am J Cardiol. 2008:102:380-5

38. Alexopoulus N, Raggi P. Epicardial Adipose Tissue: Another Tassel in the Complex Fabric of Atherosclerosis. Cardiovasc Hematol Disord Drug Targets. 2018;18:17-26.

39. Mazurek T, Zhang L, Zalewski A, et al. Human epicardial adipose tissue is a source of inflammatory mediators. Circulation. 2003;108:2460-2466.

40. Nagy E, Jermendy A, Merkely B, Maurovich-Horvath P. Clinical importance of epicardial adipose tissue. Arch Med Sci. 2017;4:864-874. 